\title{
Efecto de un programa educativo para cuidadores de personas ancianas: una perspectiva cultural
}

\section{The effect of an educational program for elderly people's caregivers: a cultural perspective}

\author{
Vilma Velásquez ${ }^{1}$, Lucero López ${ }^{1}$, Heddy López ${ }^{2}$, Nhora Cataño $^{1}$ y \\ Esperanza Muñoz ${ }^{1}$
} 1.Facultad de Enfermería. Universidad Nacional de Colombia.Bogotá. vvelasquez@bt.unal.edu.co,
allopezdi@bt.unal.edu.co, ncatano@bt.unal.edu.co, emunozt@bt.unal.edu.co

2 Universidad Católica de Colombia. Bogotá. helopd@gmail.com

Recibido 24 Agosto 2010/Enviado para Modificación 25 Mayo 2011/Aceptado 25 Julio 2011

\section{RESUMEN}

Objetivo Determinar el efecto de la aplicación de un programa educativo con perspectiva cultural para cuidadores de personas ancianas en situación de discapacidad y pobreza en un sector de Bogotá - Colombia, sobre las variables: auto percepción de salud, funcionalidad familiar, carga del cuidador y depresión.

Métodos Estudio cuasiexperimental con pre-prueba y pos-prueba. Participaron 56 cuidadores familiares, 37 en el grupo experimental (ge) y 19 en el grupo control (gc).El programa educativo aplicado al grupo experimental constaba de seis sesiones grupales con intervalo de 30 días. Cada sesión tiene cinco fases: sensibilización, información culturalmente adaptada, toma de decisiones, compromisos de acción y cierre. Fue aplicado por una enfermera experta en gerontogeriatría y enfermería transcultural. Se aplicaron las escalas de Autopercepción de Salud, Apgar familiar, Carga del cuidador y Escala de Depresión.

Resultados El programa educativo con enfoque cultural tuvo efectos significativos, sobre las variables: funcionalidad familiar $(T=2,506 ; p=0,015)$ y carga del cuidador $(\mathrm{T}=-2,149 ; \mathrm{p}=0,036)$. La autopercepción en salud y depresión no presentaron diferencias significativas.

Discusión Las acciones en educación en salud abordadas desde la perspectiva cultural abren una puerta a co-crear diversidad de terapéuticas derivadas del diálogo y la mediación entre los saberes populares y profesionales. Podría ser una clave frente al escaso éxito en la adherencia de los usuarios a las instituciones de salud y a los tratamientos.

Palabras Clave: Cuidadores, anciano, pobreza, enfermería transcultural (fuente: DeCS, BIREME). 


\section{ABSTRACT}

Objective Determining the effect of implementing an educational programme using a cultural approach for caregivers looking after elderly disabled and poor people living in a sector of Bogota, Colombia regarding the following variables: perceived health, family functioning, caregiver burden and depression.

Methods This was a quasi-experimental study involving pre-test and post-test. 56 family caregivers participated in the study, 37 in the experimental group and 19 in the control group. The educational program being applied consisted of six group sessions with a 30-day interval. Each session had five phases: awareness-raising, culturallyappropriate information, making decisions, commitments for action and closure. They were led by a nurse specializing in gerontology-geriatrics and transcultural nursing. Several scales were applied: self-perception of health, family APGAR, caregiver burden, and a depression scale.

Results The educational program involving a cultural approach had statistically significant effects on family functioning $(T=2.506 ; p=0.015)$ and caregiver burden $(p=-2.149 \mathrm{~T}=0.036)$. Self-perception of health and depression revealed no statistically significant differences.

Discussion Health education activities addressed from a cultural approach opens the way forward for creating therapeutic diversity arising from dialogue and mediation between popular and professional knowledge. It could be a key against the lack of success in users' adherence to health facilities and treatment.

Key Words: Caregiver, the aged, poverty, transcultural nursing (source: MeSH, NLM).

$\mathrm{E}$

n Colombia, como en muchos lugares del mundo, crece la población de mayores de 60 años. Los datos del último censo presentaron un incremento de 6,31\% (3 717241 ), para el 2020 se estima un aumento del 10,5\% (6 435 899) (1). El panorama imprime retos para la salud pública y la necesidad de nuevas aproximaciones para poder promover la vida digna a la población que envejece y a sus familias, principal fuente de soporte.

La discapacidad crece en el mundo a medida que las personas envejecen. La persona anciana tiene mayor riesgo de desarrollar enfermedades crónicas, siendo las causantes de morbilidad, discapacidad y mortalidad en este grupo poblacional. La pobreza tiene un efecto de mayor vulnerabilidad en las personas con discapacidad (2).

En el censo del 2005 se encontró que los mayores de 60 ocupan el 37,7 \% de la población con discapacidad (129 316) (3), 12,5\% poseen limitaciones para moverse o caminar; el 3,4\% tiene limitaciones para su autocuidado y el $2,5 \%$ posee limitaciones para hablar (4). El $40 \%$ de las personas por encima de los 85 años requieren ayuda para el desempeño de la vida cotidiana, este 
cuidado lo asumen las familias, para el 2050 en Colombia habrá un crecimiento de la población por encima de los 80 años y se proyecta que 3 millones de ancianos requerirán apoyo para el cuidado diario.

Al interior de las familias, los cuidados se asumen por el cuidador principal, quien supervisa y apoya la realización de las actividades básicas de la vida diaria como (bañar, vestir, darle de comer, entre otras actividades), apoya económicamente, participa en la toma de decisiones $(5,6)$ y no recibe retribución económica a cambio (7).

Estudios en Colombia, señalan los diversos problemas que padecen los cuidadores (8-10). El cuidador familiar de una persona con enfermedad o con discapacidad sufre cambios en todas las áreas de desempeño, como en su salud física y psicológica (11). Pasa por diversas emociones, comúnmente contrarias que le impiden mantener una sensación de bienestar (12). Algunos autores le han denominado el "síndrome del cuidador" (13).

El gran aporte del cuidador en las familias y en la sociedad, es poco apoyado por los sistemas de salud. Aunque se haya reconocido que cuidar a un familiar constituye una situación estresante (14). En Colombia, se empieza a girar la mirada hacia el cuidador y emergen importantes iniciativas a nivel político y social $(15,16)$.

El panorama revela que los cuidadores de adultos mayores con discapacidad se pueden considerar como un grupo vulnerable (17) con necesidades de promoción y prevención a los que se les debe proporcionar una adaptación a la realidad que experimentan $(18,19)$.

Además, se hace necesario que las intervenciones partan de un profundo conocimiento del contexto cultural de estas personas (19). Ofrecer un cuidado cultural, implica mediar entre el cuidado popular y el profesional que a su vez requiere del desarrollo de competencia cultural. Esto involucra, una adaptación del programa educativo de forma consistente con la cultura de los cuidadores y donde los profesionales se esfuerzan por desarrollar la sensibilidad y habilidad para trabajar efectivamente dentro del contexto cultural de las personas y la comunidad (20,21). Aproximarse a la realidad cultural de los cuidadores necesita la utilización de un lenguaje y lógica desde el discurso del cuidador (22).

A partir del enfoque mencionado, el grupo de cuidado cultural de la salud (GCCS) de la Facultad de Enfermería de la Universidad Nacional de Colombia 
ha venido desarrollando trabajos con poblaciones en múltiples condiciones de inequidad. Población anciana mestiza, afro colombiana con sus familiares, en situación de discapacidad y pobreza. En contextos rurales y urbanos-marginales. Inicialmente con estudios etnográficos fue posible el reconocimiento de los valores, creencias, factores resilientes y potencialidades que influyen el cuidado de la salud y la vida $(15,23)$. Derivado de ese conocimiento, se han generado algunas propuestas de intervención. En éste artículo presentamos los efectos del programa educativo dirigido a cuidadores familiares de personas ancianas en situación de discapacidad y pobreza (CFPADP) como una forma de responder a las demandas sociales frente a estas poblaciones y favorecer los esfuerzos que hacen las familias, en especial las mujeres, en quienes recae el peso cultural y social del cuidado de los más ancianos.

\section{METODOLOGÍA}

El estudio se realizó en Bogotá, Localidad de Kennedy, sector Patio Bonito, durante seis meses, con cuidadores familiares de personas mayores de 60 años que no pudieran salir solos de la casa, de los estratos 1 y 2 . Se utilizó un diseño de tipo cuasi experimental con preprueba y posprueba. La muestra se constituyó con 56 cuidadores, 37 en el grupo experimental (ge) y 19 en el grupo control (gc). Fueron evaluadas cuatro variables dependientes: auto percepción de salud, funcionalidad familiar, carga de cuidador y depresión.

El programa educativo estuvo dividido en seis sesiones con intervalo de 30 días y se aplicó al grupo experimental por una enfermera experta en gerontogeriatría y enfermería transcultural. Fue denominado: "Pongámosle color a la vida"; las sesiones tenían cinco fases: Sensibilización, Información Culturalmente Adaptada, Toma de Decisiones, Compromisos de Acción y Cierre. Encaminadas al auto reconocimiento, auto reflexión y el compromiso desde los propios intereses y motivaciones.

El análisis de los datos se realizó en el programa SPSS verificándose la existencia de diferencias para dos muestras independientes con la prueba $\mathrm{T}$ y se aplicó el test de Kolmogorov-Smirnov. Fueron considerados todos los cuidados éticos para un estudio de bajo riesgo de acuerdo con la resolución 8430 de 1993 del Ministerio de Salud de Colombia. Los participantes en su totalidad firmaron el consentimiento informado y al finalizar el programa para quienes requirieron de apoyo psicológico fueron remitidos a la respectiva entidad de salud. 
Instrumentos

Se creó un formato para la recolección de las características socio demográficas. El grado de dependencia de la persona anciana cuidada se evaluó con el Índice de Barthel(AVD)(24)que valora a la persona para realizar diez [10] actividades básicas de la vida diaria. La dependencia se puntúa: total [0-20], severa [2160], moderada [61-90], escasa [91-99], independencia [100].

Las variables dependientes semidieron con cuatro instrumentos: a. Encuesta de Auto percepción en salud (APS) (25) y presenta 5 opciones posibles de respuesta (excelente, muy buena, buena, regular, mala); b. El Apgar familiar tiene consistencia interna de la escalas de 0,81 y la fiabilidad test -retest de 0.81 (26). Consta de 5 preguntas en escala Likert (casi nunca, a veces, casi siempre). La funcionalidad familiar se puntúa: Normofuncional [7-10], disfunción leve [4-6] y disfunción grave [0-3]; c. Escala de carga de Zarit con consistencia interna de la escala es de 0.91 y la fiabilidad test-retest de 0.86 (27).Consta de 22 ítems en escala tipo Likert (nunca, rara vez, algunas veces bastantes veces, siempre) que puntúan entre 1 y 5 . El puntaje total $[<47]$ clasifica como no sobrecarga, entre [47 y 55] sobrecarga leve y [ $>$ a 55] sobrecarga intensa; $d$. Escala de Yesavage con validez de 0.9 y confiabilidad de 0.81 , sensibilidad de $92,7 \%$ y especificidad de $65,2 \%$ (28) tiene un formato de respuesta dicotómica (Si ó No), a las cuales se les asigna un puntaje de 0 a 1 , la prueba clasifica: $\sin$ depresión [0 a 5], depresión leve [6 a 9] o depresión establecida [> 10].

\section{RESULTADOS}

Las (os) cuidadoras (es)

Se encontró predominio de cuidadoras (75\%), en edades comprendidas entre 35 a 59 años de edad (52\%) y de 60 o más años (41\%), de estrato dos (86\%) yuno (14\%), casados (41\%) y en unión libre (21\%), con un nivel de escolaridad distribuidos así: primaria $(70 \%)$, bachillerato $(23 \%)$ sin escolaridad (5\%)y estudios superiores $(2 \%)$. En salud aventaja el régimen subsidiado $(61 \%)$ al contributivo (32\%). La HTA ocupó un $29 \%$ de las enfermedades referidas. El rol de cuidadora esta ejercido principalmente por las hijas(os) $(57 \%)$ y esposa (20\%), con más de 4 años al cuidado (79\%), distribuidos en más de 9 años (54 \%) y entre 4 y 8 años $(25 \%)$.

Las personas ancianas

El índice de Barthel reveló que el $66 \%$ se encuentran en dependencia moderada; $18 \%$ en dependencia severa; $11 \%$ en dependencia total y $5 \%$ en dependencia escasa. 
Las variables dependientes

La auto percepción en salud, en la preprueba, reveló un comportamiento semejante entre el (ge) y (gc) la mayoría de los cuidadores perciben su salud "regular" (gc) $64 \%$ y (ge) $68 \%$, como "excelente, muy buena, buena" (gc) 36 \% y (ge) $32 \%$, ningún cuidador reportó que su estado de salud fuera "malo". La pos prueba muestra que a pesar de los cambios registrados, estos no son estadísticamente significativos para la variable.

El Apgar familiar reportó disminución de la funcionalidad familiar entre la preprueba y postprueba del (gc), que pasó de 8 a 7, clasificada como Normo funcional, frente al Apgar familiar del (ge) que mantuvo el promedio en 8. En el test de Kolmogorov-Smirnov se obtuvo valores p respectivos de 0.337 y 0.22 lo que indica que las diferencias entre las pospruebas y las prepruebas del (ge) y el (gc) presentan un comportamiento normal. El valor T de 2.506 y un valor $\mathrm{p}$ de 0.015 muestran evidencia estadística suficiente para admitir que el programa educativo tuvo efecto positivo sobre la funcionalidad familiar.

La escala de carga de Zarit, conocida como carga del cuidador, mostro entre la preprueba y postprueba del (ge) una disminución de 53 sobrecarga leve a 45 no sobrecarga, frente a la puntuación promedio del $(\mathrm{gc})$ donde se conservó el promedio en 50como sobrecarga leve. Se obtuvo en el test de Kolmogorov-Smirnov un valor $\mathrm{p}$ de 0.567 y 0.250 y $\mathrm{T}$ con un valor asociado a la estadística de prueba de -2.149 y un valor $\mathrm{p}$ de 0.036 aceptándose que existe evidencia estadística suficiente para afirmar que el programa educativo incide positivamente en la reducción de la carga de los cuidadores del (ge).

La Escala de Depresión de Yesavage, reporto que los dos grupos tanto en la preprueba como en la posprueba tienen puntuaciones promedio que los clasifican "sin depresión". Se encontró que la principal diferencia que se presenta en el (ge) entre la preprueba y la posprueba es un descenso en las puntuaciones promedio de los cuidadoresde 4 a 3 clasificado "sin depresión". Lo que demuestra que los valores medios de las pospruebas del (gc) y del (ge) no difieren significativamente de acuerdo a lo encontrado con el test de KolmogorovSmirnov donde se obtuvo un valor $\mathrm{p}$ de 0.087 y 0.219 y $\mathrm{T}$ con un valor asociado a la estadística de prueba de -0.062 y un valor $\mathrm{p}$ de 0.951 , aceptándose que no existe evidencia estadística suficiente para admitir que el programa educativo tenga efecto positivo sobre el comportamiento de la escala de Yesavage. 


\section{DISCUSIÓN}

Los resultados reflejan una proporción mayor de mujeres desempeñando el rol de cuidadoras y cuyos familiares presentan en su mayoría un grado de dependencia moderada. Así, el perfil de la cuidadora familiar corresponde al de una mujer de edad media (35-60 años), ama de casa, descendiente de primer grado de la persona cuidada (principalmente hija) o cónyuge, con un nivel de escolaridad bajo, estos hallazgos reportan semejanza con diversos estudios del ámbito nacional e internacional (29-31).

El programa educativo dirigido a CFPADP con enfoque cultural tiene efectos estadísticamente significativos sobre las variables: carga del cuidador y funcionalidad familiar. El efecto positivo en estas variables tiene directa relación con la concepción del programa donde se proporciona un entorno de confianza, de interacción con los valores y creencias de los participantes por medio de palabras, dichos y símbolos de la cotidianidad. Además, se proporciona un ambiente lúdico adaptado a su cosmovisión que favorece la construcción de redes de afecto y empatía entre los cuidadores. El profesional sostiene una relación horizontal, de constante reconocimiento del rol que desempeñan, de promoción del auto reconocimiento de capacidades y de valoración de los esfuerzos de los cuidadores.

La conjugación de las temáticas propuestas, de las cinco fases y las actividades con simbolismo cultural encaminadas al descubrimiento grupal de las fortalezas como personas y cuidadores, al establecimiento de límites en la familia, al auto establecimiento de metas y compromisos a desarrollar en la vida cotidiana, de acuerdo con la situación particular, contribuyeron a la mejora en las dos variables.

No se reveló un efecto especial sobre la auto percepción de salud y la depresión. La auto percepción de la salud aunque el $64 \%$ (gc) y $68 \%$ (ge) perciben la salud como regular, ningún cuidador reportó su salud como mala. Dichos resultados están permeados por un fenómeno cultural detectado en los trabajos etnográficos previos del(GCCS) y durante el desarrollo de las sesiones. Los cuidadores se ven a sí mismos como seres fuertes, casi invencibles porque sienten sobre sus hombros la vida y salud de la persona a quien cuida. Esta actitud de fortaleza los pone en una situación de riesgo, confirmada en las sesiones, donde se les pedía que sobre una figura del cuerpo colocaran puntos rojos donde tenían malestar ó dolor. La mayoría puso en evidencia sus 
enfermedades físicas pero tuvieron oportunidad de compartir prácticas populares y profesionales para atender esas problemáticas.

La variable depresión, llamó la atención puesto que las puntuaciones medias indican que los dos grupos se encuentran "sin depresión". La literatura internacional muestra una problemática emocional de los cuidadores y los estudios preliminares del (GCCS) mostraban que ancianos están cuidando de ancianos y sus problemas de "penas" denominación popular a los problemas afectivos, tal vez, el resultado pudo afectarse debido a que el $52 \%$ de los cuidadores se encontró en edades inferiores a 60 años y el instrumento aplicado es particular para población anciana. Futuros estudios deberán considerar instrumentos que puedan ser útiles para diversas edades.

En general, la literatura internacional reporta diversas propuestas de intervención hacia los cuidadores y las propuestas educativas tienen una marcada predilección. Sin embargo, las propuestas analizadas dan poca relevancia a los aspectos de los valores y creencias de los participantes. Es escasa la evidencia de estudios donde se evalúe los efectos de programas o actividades educativas sobre los cuidadores, sin embargo, la eficacia de estas intervenciones radica en el consejo o instrucción a los cuidadores para solucionar problemas $(32,33)$.

Los efectos del programa educativo con una perspectiva cultural, son un eslabón ante la necesidad de construir mayor evidencia frente a los efectos de las acciones que promueven los diversos profesionales con el grupo de cuidadores familiares. Consideramos, que los efectos mostrados son indicador de la necesidad de hacer un reconocimiento de la dimensión cultural como eje que acciona un mundo colectivo e individual donde, los cuidadores ganan el estatus de colaboradores mediante procesos de auto reconocimiento y autodeterminación mediada por lo que le es significativo en su entorno, por el establecimiento de sus propias metas y acciones acorde con sus capacidades.

Los profesionales ganan porque enriquecen su universo práctico y profesional al descubrir que la creatividad y la negociación entre saberes se hace prioritaria para construir propuestas congruentes con el universo cotidiano de los usuarios de los servicios de salud. Ésta podría ser una clave para combatir el escaso éxito en la adherencia de los usuarios a las instituciones de salud y a los tratamientos.

Conviene incentivar trabajos investigativos con ésta perspectiva donde participen diversos profesionales y grupos en diferentes áreas rurales, urbanas, 
niveles socioeconómicos y culturales. Se necesita tener cuidado cuando se trabaje con poblaciones de bajos recursos porque la permanencia en los programas se ve afectada por la imposibilidad del cuidador de encontrar a otra persona que lo remplace o por la necesidad de salir a buscar recursos económicos para el sostenimiento familiar. El aporte práctico e investigativo hacia el grupo de cuidadores de personas ancianas es una inversión social de impacto en la salud pública que requiere una construcción de políticas públicas $\mathrm{y}$ acciones de los profesionales en salud que visibilice, proteja y apoye a los cuidadores

Agradecimientos: Al equipo directivo y de profesionales del Centro de Desarrollo Comunitario Bellavista en Bogotá por el apoyo locativo y en la convocatoria de los participantes. Trabajo enmarcado en los proyectos del grupo de investigación en Cuidado Cultural de la salud, con el apoyo financiero de la División de Investigación Bogotá (DIB) de la Universidad Nacional de Colombia, 2008 - 2010 código 8007031.

\section{REFERENCIAS}

1. Departamento Administrativo Nacional de Estadística (DANE) (2008). Censo general 2005. Nivel Nacional. [Internet]. Disponible en: http://www.dane.gov.co/censo/files/ libroCenso2005nacional.pdf Consultado en Marzo de 2010.

2. Ruipérez I. Envejecimiento, siglo XXI y solidaridad. Esp. Geriatr Gerontol 2002; 37(Suppl 2):3-7.

3. Departamento Administrativo Nacional de Estadística (DANE) (2007). Discapacidad. Personas con limitaciones permanentes. [Internet]. Disponible en: http://www.dane.gov.co/ censo/files/discapacidad/preva_indices.pdf Consultado en Diciembre de 2009.

4. Ministerio de la protección Social. Diagnóstico preliminar sobre personas mayores, dependencia y servicios sociales en Colombia. [Internet]. Disponible en: http:// www.minproteccionsocial.gov.co/asistenciaSocial/Documents/ Situacion\%20Actual\%20de\%20las\%20Personas\%20adultas\%20mayores.pdf?Mobile=1 \&Source $=\% 2$ FasistenciaSocial\%2F_layouts $\% 2 F m o b i l e \% 2 F v i e w . a s p x \% 3 F L i s t \% 3 D 49$ 70a592-fb81-411f-b845-f61b04dce501\%26View\%3D5d7fbd7c-b41f-4463-888620c8385a07fe\%26CurrentPage\%3D1Consultado en Febrero de 2010.

5. Hortelano P, Zapata MA, Fernández E. Sentimientos y emociones en cuidadores informales. Hygia. 2010; 1er Cuatrimestre (73):47-53.

6. Pinto N, Barrera L, Sánchez B. Reflexiones sobre el cuidado a partir del programa: Cuidando a los cuidadores. Aquichán. 2005; 5(1):128-137.

7. Monleón M, Dávals RK, Polo A, Villacañas M. Perfil sociocultural del cuidador principal de la persona mayor dependiente y su formación específica en cuidados. Enfermería Comunitaria. 2007; 3(1):20-25.

8. Barrera L, Pinto N, Sánchez B. Cuidando a los Cuidadores. Un programa de apoyo a fa familiares de personas con enfermedad crónica. Index de Enfermería [Internet]. Disponible en: http://www.index-f.com/index-enfermeria/52-53/5458.php Consultado en Junio de 2010.

9. Montalvo A, Flórez IE. Cuidadores de Cartagena y su contexto de cuidado: estudio comparativo. Aquichán. 2009; 9(2):135-146.

10. Giraldo CI, Franco GM, Correa LE, Salazar MO, Tamayo AM. Cuidadores familiares de ancianos: ¿quiénes son y cómo asumen este rol?. Facultad Nacional de Salud Pública $2005 ; 23(2): 7-15$. 
11. Espín AM. Escuela de Cuidadores como programa psicoeducativo para cuidadores informales de adultos mayores con demencia. Cubana de Salud Pública 2009; 35(2):1-14.

12. Pinto $\mathrm{N}$. El reto de los cuidadores familiares de personas en situación crónica de enfermedad. Cuidado y práctica de enfermería. Bogotá: Unibiblos; 2000. p. 172-183.

13. Pérez JM, Abanto J, Labarta J. El síndrome del cuidador en los procesos con deterioro cognoscitivo (demencia). Aten Primaria. 1996; 18(4):194-202.

14. De la Cuesta C. El cuidado familiar: una revisión crítica. Investigación y educación en enfermería. 2009; 27(1):96-102.

15. Cárdenas A, López AL. Analysis Matrix of Resilience in the Face of Disability, Old Age and Poverty. International Journal of Disability, Development and Education. 2010; 57(2): 175-189.

16. López AL. Cuidando al adulto y al anciano. Aquichán. 2003; 3(3): 52-58.

17. Velásquez $\mathrm{V}$. Caracterización del riesgo familiar total en familias con adultos mayores con discapacidad, Patio Bonito, Localidad Kennedy, Bogotá, 2005. Avances en Enfermería. 2009; 27(1):69-81.

18. Dueñas E, Martínez MA, Morales B, Muñoz C, Viáfara AS, Herrera JA, et al. Síndrome del cuidador de adultos mayores discapacitados y sus implicaciones psicosociales. Colombia Médica. 2006; 37(Supl 1):31-38.

19. Villarreal M, Dimmitt J, Salazar BC. Experiencias Cotidianas de Cuidadoras de Personas con la Enfermedad de Alzheimer. Desarrollo Cientif. Enferm. 2006; 14(9): 342-347.

20. Purnell L. The Purnell model for cultural competence. Transcultural Nurs. 2002; 13: 193-196.

21. Campinha J. Cultural Competence in Psychiatric Nursing: Have You "ASKED" the Right Questions?. Journal of the American Psychiatric Nurses Association. 2002; 8: 183187.

22. Vila J, Garriga R. La problemática de los cuidadores mayores. Informaciones Psiquiátricas. 2000; 0(162):363-371.

23. Mejía LD, López AL, Velásquez V. Hierbas medicinales: un asunto más allá de las prácticas populares. Avances en el Cuidado de Enfermería. Bogotá: Universidad Nacional de Colombia; 2010; p. 383-401.

24. Ruzafa J, Moreno J. Valoración de la Discapacidad Física: El índice de Barthel. Revista Española de Salud Pública. 1997; 71: 177-187.

25. Centro Latinoamericano y Caribeño de Demografía (CEPAL). Manual Sobre Indicadores de Calidad de Vida en la Vejez. Santiago de Chile. 2006. [Internet]. Disponible en: http:// www.eclac.org/publicaciones/xml/0/28240/W113 1.pdf Consultado en Mayo de 2010.

26. Bellón JA, Delgado A, Luna J, Lardelli P. Validez y fiabilidad del cuestionario de función familiar Apgar-familiar. Atención primaria. Sociedad Española de medicina de Familia y Comunitaria. 1996; 18(6):289-296.

27. Gallud J, Guirao G, JosepA. Escalas e instrumentos para la valoración en atención domiciliaria. Valencia: Generalitat Valenciana. Conselleria de Sanitat; 2004.

28. Almeida O, Almeida S. Short versions of the geriatric depression scale: a study of their validity for the diagnosis of a major depressive episode according to ICD-10 and DSM-IV. International Journal of Geriatric Psychiatry. 1999; 14: 858-865.

29. Badia X, Lara N, Roset M. Calidad de vida, tiempo de dedicación y carga percibida por el cuidador principal informal del enfermo de Alzheimer. Aten Primaria. 2004; 34:170177.

30. Montalvo A, Florez IE, Stavro D. Cuidado A Cuidadores Familiares De Meninos em Situação de Discapacidade. Aquichán. 2008; 8(2):197-211.

31. Barrera L, Blanco L, Figueroa P, Pinto N, Sánchez B. Habilidad de cuidadores familiares de personas con enfermedad crónica. Mirada internacional. Aquichán. 2006; 6 (1):22-33.

32. Díaz JC, Rojas MV. Cuidando al cuidador: efectos de un programa educativo. Aquichán. 2009; 9(1):73-92.

33. Sorensen PD. How effective are interventions with caregivers? An update meta-analysis. The Gerontologist 2002; 42(3):356-72. 